\title{
Desenvolvimento de Mato Grosso do Sul: uma análise dos setores produtivos do estado a partir do modelo insumo- produto
}

\author{
Edrilene Barbosa Lima Justi \\ Universidade Federal de Mato Grosso do Sul - Nova Andradina - Mato Grosso do \\ Sul - Brasil \\ ORCID: http://orcid.org/0000-0003-0281-2603 \\ Raul Asseff Castelao \\ Universidade Anhanguera-Uniderp - Campo Grande - Mato Grosso do Sul - \\ Brasil \\ ORCID: http://orcid.org/0000-0002-4677-6088

\section{Celso Correia de Souza} \\ Universidade Anhanguera-Uniderp - Campo Grande - Mato Grosso do Sul - \\ Brasil \\ ORCID: http://orcid.org/0000-0002-2689-8264

\section{Daniel Massen Frainer} \\ Universidade Estadual de Mato Grosso do Sul - Campo Grande - Mato Grosso do \\ Sul-Brasil \\ ORCID: http://orcid.org/0000-0003-0813-214x
}

\section{Resumo}

Este trabalho visou analisar, a partir da matriz de insumo-produto, indicadores do grau de interligação setorial da economia de Mato Grosso do Sul (MS) e impactos de demanda sobre emprego e renda. Este modelo de equilíbrio geral permite que políticas de geração de emprego e renda, necessárias para o desenvolvimento do Estado, se baseiem em uma proposta voltada à Sociedade, ao Ambiente e ao Desenvolvimento Regional Sustentável. Sendo assim, os setores que mais impactam a economia do Estado foram assim identificados: Comércio e Serviços, Celulose e Produtos de Papel, Extrativa Mineral, Artigos do Vestuário e Acessórios e Artefatos de Couro. Os resultados apresentados servem de orientação para a elaboração e planejamento racional de políticas públicas e privadas que visem estimular o crescimento econômico do Estado.

Palavras-chave: Matriz Insumo-Produto. Multiplicadores de Impacto. Índices de Ligação. Mato Grosso do Sul. 


\section{Mato Grosso do Sul development: an analysis of the productive sectors using the input- output model}

\section{Abstract}

This work aims to analyze, from the input-output matrix, sectoral interconnection level indicators of Mato Grosso do Sul (MS) economy and demand impacts on employment and income. This general equilibrium model allows to generate employment and income policies, necessary for the development of the State based on a proposal directed to the Society, the Environment and Sustainable Regional Development. Thus, the sectors that impact the most were identified as follows: Trade and Services, Pulp and Paper Products, Mining and Quarrying, Clothing Items and Accessories, Leather Goods. The results presented provide guidance for the development and rational planning of public and private policies to stimulate the State's economic growth.

Keywords: Input-Output Matrix. Impact Multipliers. Linkage Indices. Mato Grosso do Sul.

\section{Desarrollo de Mato Grosso do Sul: un análisis de los sectores productivos del estado utilizando el modelo input-output}

\section{Resumen}

Este trabajo tuvo como objetivo analizar, a partir de la matriz input-output, indicadores del grado de interconexión sectorial de la economía de Mato Grosso do Sul (MS) y los impactos de la demanda en el empleo y los ingresos. Este modelo de equilibrio general permite que las políticas de empleo y generación de ingresos, necesarias para el desarrollo del Estado, se basen en una propuesta dirigida a la Sociedad, el Medio Ambiente y el Desarrollo Regional Sostenible. Por lo tanto, los sectores que más impactan en la economía del Estado se identificaron de la siguiente manera: Comercio y servicios, productos de pulpa y papel, extracción de minerales, ropa y accesorios y artículos de cuero. Los resultados presentados sirven como guía para la elaboración y planificación racional de políticas públicas y privadas que tienen como objetivo estimular el crecimiento económico del Estado.

Palabras clave: Matriz de entrada-producto. Multiplicadores de impacto. Índices de conexión. Mato Grosso del Sur.

\section{Introdução}

A sociedade humana tem evoluído à medida que se esforça por melhorar sua condição de subsistência. Isso inclui a busca constante por criar meios para suprir suas diversas necessidades e, em alguns aspectos, o processo é tão sofisticado que o simples suprimento básico se transforma em desejos por produtos e serviços específicos. Pesquisas são desenvolvidas, instituições são criadas e inovações são almejadas a ponto de tornar o ambiente em que vivemos um lugar marcado por constantes mecanismos de trocas ou transações entre pessoas, físicas e/ou jurídicas.

Nesse sentido, um bem pode ser considerado qualquer objeto material que possa atender às necessidades das pessoas e do mercado no ambiente em que interagem compradores e vendedores dos bens ou serviços, transformados em mercadorias (BÊRNI et al., 2011).

Esse modelo de trocas é denominado de fluxo circular da atividade econômica, em que organizações econômicas interagem constantemente moldando as sociedades, ora promovendo desenvolvimento e crescimento ora promovendo retrocessos. São os produtores ou empresas, fatores de produção ou famílias (THOMAS e CALLAN, 2010; BÊRNI et al., 2011). 
Sendo assim, as sociedades estimulam o aprimoramento de suas atividades econômicas, visando a continuidade das trocas de mercadorias para garantir a satisfação de suas necessidades e desejos. E nesse contexto, preocupa-se em melhorar os processos de produção, distribuição, circulação e consumo dos bens e serviços.

Considerando um cenário regional, pode-se aplicar essa sistemática ao estado de Mato Grosso do Sul (MS), cujo desenvolvimento econômico tem destaque para vocação na agropecuária, grande setor do MS que corresponde a 18\% de seu Produto Interno Bruto (PIB) e de 5\% de participação do setor no PIB do Brasil (IBGE, 2015).

Mato Grosso do Sul é um dos 27 Estados da federação brasileira e possui, aproximadamente, 36 milhões de hectares, com população estimada em 2,7 milhões de habitantes e 79 municípios em sua divisão territorial (IBGE, 2020). A qualidade do clima e água existente no Estado são propicias às diversas atividades do agronegócio tendo predominância de pastagens nativas (Bungenstab, 2012).

Entre os anos de 1991 a 2010, o Estado passou de 72 municípios (1991) para 78 em 2010 e, com a criação de Paraíso das Águas em 2013, tem atualmente 79 municípios. Conforme o caderno estatístico da Secretaria de Estado de Meio Ambiente e Desenvolvimento Econômico (SEMADE), a população de MS apresenta um crescimento médio de 17\%, levando-se em consideração 1991 como ano base.

Com a análise da série do desempenho do PIB do Estado, de 2010 a 2013, verificou-se um crescimento a uma taxa média de 5\%, enquanto o PIB nacional obteve um crescimento em torno de $3 \%$ ao ano (IBGE, 2015). O PIB do MS representa $14 \%$ do total do centro oeste e 1,46\% do PIB brasileiro. Em relação à composição do seu Produto Interno Bruto, a atividade terciária representa aproximadamente $60 \%$, $22 \%$ setor secundário e o setor primário $18 \%$. Contudo, em 2017, a agropecuária Sul-mato-grossense cresceu 25,0 \%. O segundo setor, que representa as atividades ligadas à indústria cresceu $1,5 \%$ e o setor terciário apresentou redução -0,4\% (SEMADE, 2015).

Coutinho et al. (2019) aponta que as principais atividades na criação de emprego foram do setor de serviços (administração pública, serviços e comércio), no entanto, as atividades de indústria e ligadas ao agronegócio passam a ter destaque nos últimos anos sendo, portanto, importante indutores de emprego e renda na economia regional.

Fagundes et al. (2017) descreve que o Estado tem se destacado no cenário nacional e também internacional em função as suas características propicias a produção agroindustrial e pela oportunidade na possibilidade de pioneirismo no desenvolvimento de novas tecnologias como, por exemplo, biodiesel.

Assim, surge a questão que norteou este trabalho: como se comportam os agentes econômicos no Mato Grosso do Sul em um contexto recente da economia regional? Para tanto foi desenvolvida estimativas a partir da estrutura da economia regional considerando 20 setores econômicos pelo método de análise da matriz insumo-produto regional de Mato Grosso do Sul.

Com o objetivo geral de analisar os setores produtivos do Estado, foram estimados vários indicadores a partir da matriz de insumo-produto buscando entender as relações intersetoriais da economia, além de suas relações com o resto do Brasil e do Mundo. Os objetivos específicos elencados para atingir o objetivo 
geral foram: i) identificar nas transações entre setores produtivos, quais os que mais impactam a economia de Mato Grosso do Sul, em um cenário de aumento de demanda por bens e serviços finais; ii) apontar os impactos em termos de geração de empregos no Mato Grosso do Sul e; iii) verificar os impactos em termos de geração de renda no Mato Grosso do Sul, em um cenário de aumento de demanda nos produtos finais dos setores produtivos estudados.

O presente estudo está estruturado, além da presente introdução, da seguinte maneira: 2 ) revisão de literatura, aonde se aborda a questão das demandas das sociedades e suas externalidades; 3) Material e Métodos, aonde se tem elencadas as fontes de dados, o método de estudo realizado e as análises realizadas; 4) no tópico Resultados e Discussão está contida a discussão em torno dos resultados obtidos; 5) a seguir, as conclusões, com os principais destaques encontrados e, por fim, o tópico final com as literaturas utilizadas.

\section{Revisão de Literatura}

O estudo das relações de troca de uma dada sociedade/economia por meio da Matriz Insumo-Produto é amplamente utilizado em vários países e regiões. A base do estudo da MIP deriva dos estudos de François Quesnay (1758), a Tableu de Economique. Contudo, esta técnica ganhou amplitude com os trabalhos de Leontief (1936) e, a partir disso, se tornou instrumento quase que obrigatório no estudo das relações intersetoriais em uma economia ou considerando a relação de duas ou mais economias (CASTELAO et al., 2019).

No Brasil, diversos estudos foram desenvolvidos com base na MIP, destacando as matrizes desenvolvidas pelo IBGE para a economia nacional a cada cinco anos e os trabalhos de Miranda (1980), Guilhoto (1986), Montoya (2001) e Haddad et al. (2017).

Miranda (1980) descreve de forma específica os impactos sobre o meio ambiente e a atividade industrial do médio rio Paraíba do Sul utilizando a Matriz Insumo-Produto. A conclusão do estudo foi de que entre os anos de 1978-1982, as atividades industriais naquela localidade não afetavam de forma demasiada a qualidade da água.

Gonçalves Junior e Guilhoto (2014) analisaram, a partir da MIP, a estrutura produtiva e as relações comerciais do estado do Paraná na economia brasileira. Com base nesse estudo, concluíram que o Paraná possui uma forte relação comercial industrial com outros estados do Brasil, em especial determinadas regiões do Paraná.

Costa et al. (2013), no trabalho "Importância dos Setores Agroindustriais na Geração de Renda e Emprego para a Economia Brasileira" e concluem que ao empregarem a MIP como técnica de análise econômica, os choques em setores da agroindústria obtiveram melhor desempenho se comparado aos choques feitos em setores industriais.

Além do uso da MIP para economias de país ou de estado, é possível também desenvolver estudos em regiões metropolitanas como, por exemplo, estudo feito por Castelani et al. (2013) o qual objetivou estimar quanto do desmatamento na Amazônia se deve ao consumo de bens e serviços de famílias que 
vivem na própria região amazônica, comparando-o ao desmatamento causado por consumidores que vivem fora da Amazônia.

Haddad et al. (2018) simularam o impacto de diferentes tipos de investimentos em mobilidade na região metropolitana de São Paulo através do uso da MIP e fizeram 10 cenários diferentes para avaliar o impacto dos investimentos na cadeia produtiva regional metropolitana e como isso afeta a estrutura de emprego e renda.

De forma concisa, o estudo de desenvolvimento econômico dos Estados brasileiros e, inclusive do próprio país, se apresenta com farta literatura quando do uso da matriz insumo-produto como ferramenta de análise. Cabe ressaltar ainda a existência de trabalhos que procuraram descrever a relação entre a economia e o meio ambiente em escala regional para os estados de Pernambuco, Bahia, Rio Grande do Sul, São Paulo e Mato Grosso do Sul (SALVO et al., 2015; MONTOYA et al., 2013; MONTEIRO et al., 2012).

\section{Elementos Teóricos e Metodológicos}

O método utilizado na estimativa da matriz de insumo-produto foi originalmente desenvolvido por LEONTIEF (1986), em que foi estimado de forma empírica as inter-relações entre os diferentes setores da economia NorteAmericana, revelando através da covariação de preços, produções, investimentos e rendas, como o país se relacionava com o mundo, interpretando todas as suas relações em termos de transações monetárias de bens e serviços.

De acordo com MILLER e BLAIR (2009), um modelo de insumo-produto é construído a partir de dados observados em um contexto econômico, podendo ser um modelo que represente determinada área, como uma nação, por exemplo. A atividade econômica deve ser capaz de ser separada em um número de segmentos ou setores que compõe a estrutura de produção da economia. Os dados necessários são fluxos de produtos, mensurados em unidades monetárias ou não, de cada um dos setores (como produtor/vendedor), para cada um dos setores (como um comprador). Estes fluxos interindustriais, ou transações, ou mesmo, fluxos intersetoriais são denominados de análise insumo-produto (input-output analysis), e normalmente são representados por determinado período (geralmente um ano).

As relações do modelo de insumo-produto são representadas, de modo esquemático matricial na figura 1. De acordo com ela, pode-se observar que as vendas de um dado setor são utilizadas como insumo no processo produtivo de outro setor ou podem também ser consumidas pelos vários componentes da demanda final. De outro lado, observando-se as colunas nota-se que o processo produtivo de um determinado setor exige uma determinada quantidade de insumos que podem ser originados da própria economia ou importados. Este mesmo processo produtivo é responsável tanto pelo pagamento de impostos quanto pela geração de valor adicionado, na forma de geração de salários e de excedentes. 
Figura 1. Relação esquemática matricial do modelo de insumo-produto

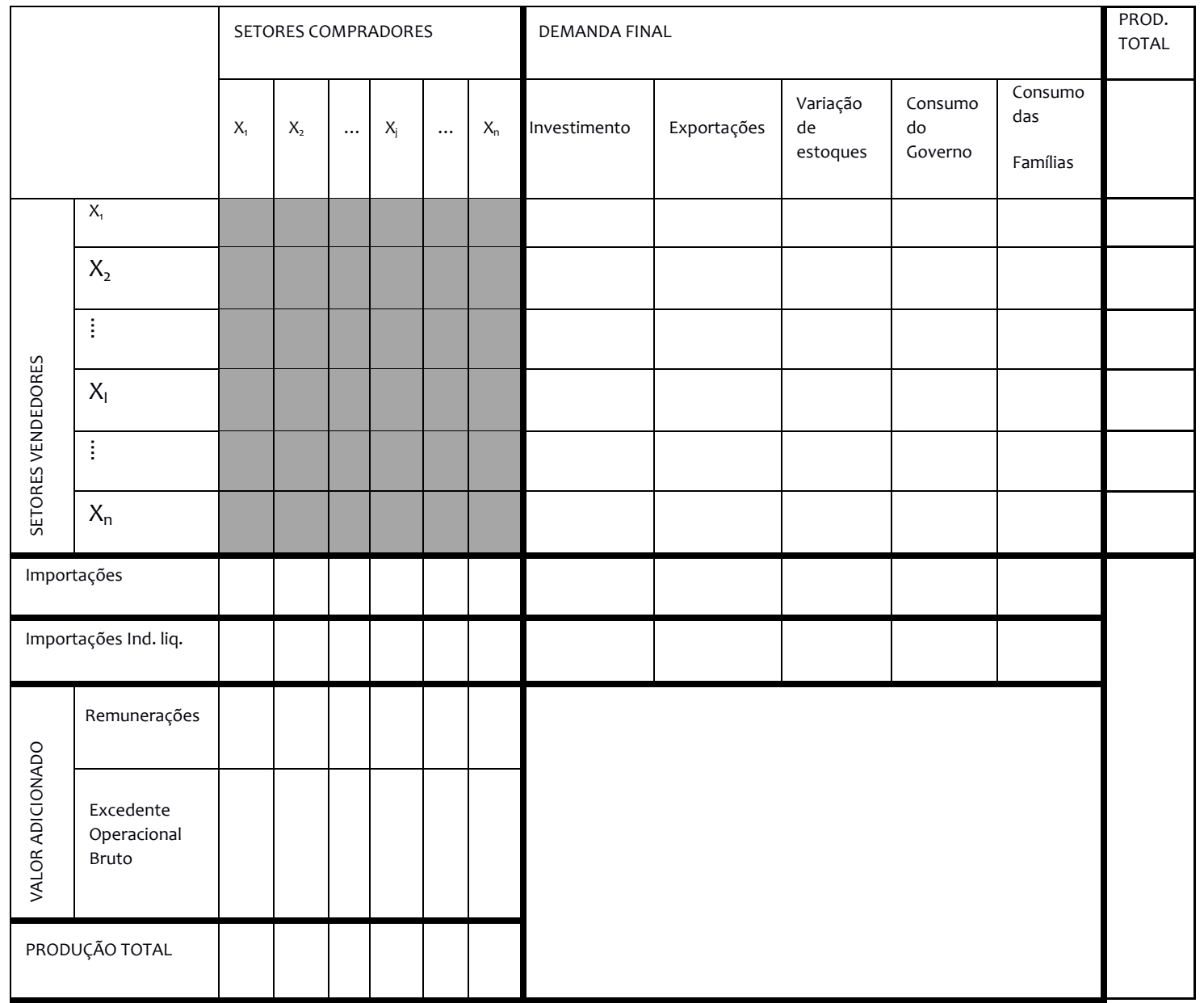

Fonte: Adaptada de Hilgemberg (2004).

Na figura 1, a demanda de um dado setor $j,(j=1,2,3, \ldots, n)$, por insumos originados de outros setores é relacionada com o montante de bens produzidos por este mesmo setor j e a demanda final, isto é, a demanda das famílias, do governo ou de outros países (exportações), é determinada por considerações não relacionadas com o montante produzido nestas unidades (MILLER e BLAIR, 2009).

Deste modo, assumindo que a economia é dividida em $n$ setores, tem-se o modelo representado na equação (1).

$$
\sum_{j=1}^{n} z_{i j}+C_{i}+G_{i}+I_{i}+E_{i}=X_{i} \quad i=1,2,3, \cdots, n
$$

Onde $z_{i j}$ é a produção do setor $i$ utilizada como insumo intermediário pelo setor $j ; C_{i}$ é a produção do setor i consumida pelas famílias; $I_{i}$ é a produção do setor $i$ destinada ao investimento; $G_{i}$ é a produção do setor $i$ destinada às administrações públicas; $E_{i}$ é a produção do setor i exportada e; $X_{i}$ é a produção total do setor $i$ (demanda final e insumos intermediários). 
A demanda total de produção do setor i, denotada por $Y_{i}$, equação (2), é obtida pelo somatório das produções do setor i que são demandadas pelas famílias, pelo governo, para investimentos e para exportações.

$$
Y_{i}=C_{i}+G_{i}+I_{i}+E_{i}
$$

O modelo de insumo-produto assume que os fluxos interindustriais do setor $i$ para o setor j obedecem a uma relação exata, dada por coeficientes técnicos $a_{i j}$, que expressam a quantidade de insumos do setor i necessária à produção de uma unidade de produto do setor j, e é definido pela equação (3).

$$
a_{i j}=\frac{z_{i j}}{X_{j}} \quad i=1,2,3, \cdots, n ; j=1,2,3, \cdots, n
$$

Onde $X_{j}$ é a produção total do setor $j$.

O conjunto de coeficientes técnicos $\mathrm{a}_{\mathrm{ij}}(i, j=1,2,3, \cdots, n)$ forma uma matriz $A$ de dimensão $n \times n$, que postulam medidas fixas das relações entre a produção de um setor e seus insumos.

Isolando $z_{i j}$ na equação (3) obtém-se $z_{i j}=a_{i j} X_{j}$ que, substituído em (1), juntamente com a equação (2), obtém-se a equação (4).

$$
\sum_{j=1}^{n} a_{i j} X_{j}+Y_{i}=X_{i} \quad i=1,2,3, \cdots, n
$$

Desenvolvendo a equação (4), para $(i, j=1,2,3, \cdots, n)$, obtém-se o sistema de equações (5).

$$
\left\{\begin{array}{ccccc}
a_{11} X_{1}+a_{12} X_{2}+ & \cdots & a_{1 n} X_{n}+Y_{1} & = & X_{1} \\
a_{21} X_{1}+a_{22} X_{2}+ & \cdots & a_{2 n} X_{n}+Y_{2} & = & X_{2} \\
a_{31} X_{1}+a_{32} X_{2}+ & \cdots & a_{3 n} X_{n}+Y_{3} & = & X_{3} \\
\cdots & \cdots & \cdots & \cdots & \cdots \\
a_{n 1} X_{1}+a_{n 2} X_{2}+ & \cdots & a_{n n} X_{n}+Y_{n} & = & X_{n}
\end{array}\right.
$$

Isolando-se os valores de $Y_{1}, Y_{2}, Y_{3}, \ldots, Y_{n}$ e colocando os fatores comuns em evidência, obtém-se o sistema matricial aberto de Leontief, representando a proporção fixa dos insumos por unidade do produto final, modelo (6).

$$
\left[\begin{array}{ccccc}
1-a_{11} & -a_{12} & -a_{13} & \cdots & -a_{1 n} \\
-a_{21} & 1-a_{22} & -a_{23} & \cdots & -a_{2 n} \\
-a_{31} & -a_{32} & 1-a_{33} & \cdots & -a_{3 n} \\
\cdots & \cdots & \cdots & \cdots & \cdots \\
-a_{n 1} & -a_{n 2} & -a_{n 3} & \cdots & 1-a_{n n}
\end{array}\right]\left[\begin{array}{c}
X_{1} \\
X_{2} \\
X_{3} \\
\cdots \\
X_{n}
\end{array}\right]=\left[\begin{array}{c}
Y_{1} \\
Y_{2} \\
Y_{3} \\
\cdots \\
Y_{n}
\end{array}\right]
$$

A representação matricial do sistema aberto de Leontief (6), para os $n$ setores que compõem a economia, é dada matricialmente pela equação (7).

$$
(I-A) X=Y
$$


Sendo,

$$
A=\left[\begin{array}{ccccc}
a_{11} & a_{12} & a_{13} & \cdots & a_{1 n} \\
a_{21} & a_{22} & a_{23} & \cdots & a_{2 n} \\
a_{31} & a_{32} & a_{33} & \cdots & a_{3 n} \\
\cdots & \cdots & \cdots & \cdots & \cdots \\
a_{n 1} & a_{n 2} & a_{n 3} & \cdots & a_{n n}
\end{array}\right] \quad ; \quad X=\left[\begin{array}{c}
x_{1} \\
x_{2} \\
x_{n} \\
\cdots \\
x_{n}
\end{array}\right] \quad \text { e } \quad Y=\left[\begin{array}{c}
y_{1} \\
y_{2} \\
y_{n} \\
\cdots \\
y_{n}
\end{array}\right]
$$

Isolando-se $X$ da equação (7), obtém-se a produção total $X$ necessária para suprir a demanda final $Y$, equação (8).

$$
X=(I-A)^{-1} Y
$$

Onde $(I-A)^{-1}$ é a matriz de coeficientes técnicos diretos e indiretos, denotada por $Z$, também conhecida como matriz de Leontief ou matriz inversa de Leontief, a qual indica os requerimentos diretos (provenientes da demanda final) e indiretos (provenientes da demanda intermediária) para a produção de bens e serviços na economia, isto é, cada elemento da matriz corresponde aos requisitos diretos e indiretos da produção total do setor i necessários para produzir uma unidade de demanda final do setor $j$.

A partir dos coeficientes diretos e da matriz inversa de Leontief é possível estimar, para cada setor da economia, o quanto é gerado direta ou indiretamente de emprego, renda e valor adicionado para cada unidade produzida para a demanda final. Pode-se ainda estimar os multiplicadores de impacto que medem a cada aumento unitário na demanda final qual o resultado sobre o valor adicionado, o emprego, os rendimentos ou qualquer outra variável apresentada na matriz na forma de vetor linha (FEIJÓ, 2013).

Dessas análises, tem-se o modelo fechado da matriz, que pode ser utilizado para mensurar os efeitos diretos, indiretos e induzidos sobre a geração de emprego e renda nos setores a partir de alterações nas variáveis de demanda final, onde pela matriz de Leontief tem a capacidade de gerar coeficientes que endogeneizam o consumo das famílias, permitindo medir os efeitos multiplicadores desse consumo sobre o restante da economia (MILLER e BLAIR, 2009; GUILHOTO, 2011).

\subsection{Medidas derivadas: indicadores síntese}

Esses indicadores foram desenvolvidos procurando sintetizar a informação contida nas matrizes de coeficientes técnicos. Os indicadores síntese ou índices de ligações para frente e para trás, forward e backward linkages, identificam os setoreschave na economia. São, também, denominados índices de Hirschman-Rasmussen e indicam o grau de encadeamento dos setores da economia, tanto para trás como para frente, ou seja, evidenciam o grau com que um setor demanda ou oferta insumos para os demais setores do sistema econômico.

Os valores calculados para os índices de ligações para trás indicam quanto o setor demanda de outros setores da economia, enquanto os índices de ligações para frente mostram o quanto o setor é demandado pelas outras indústrias. De outra forma, diz-se que estes índices medem o encadeamento entre os setores 
considerados na matriz de Leontief (RASMUSSEN 1956; HIRSCHMAN, 1958; GUILHOTO et al., 2002; FEIJÓ, 2013).

O índice de ligação para frente, foward linkage ( $F L)$, de um setor i também pode ser interpretado como sendo o aumento total da produção de todos os setores da economia quando há um aumento unitário pela demanda final do setor $i$, equação (9).

$$
F L_{i}=\sum_{j=1}^{n} z_{i j} \quad \rightleftarrows \quad i=1,2, \cdots, \cdots n
$$

Quanto ao índice de ligação para trás, backward linkage (BL), este pode ser visto como sendo o aumento da produção do setor $j$ quando acontece um aumento unitário na demanda final da economia, equação (10).

$$
B L_{j}=\sum_{i=1}^{n} z_{i j} \quad \rightleftarrows \quad j=1,2, \cdots, n
$$

Para comparações de matrizes são desenvolvidos índices normalizados, ou seja, calcula-se para cada linha ou coluna da matriz de Leontief a relação entre o seu coeficiente médio e a média total dos coeficientes.

\subsection{Multiplicadores de impacto}

Os multiplicadores de impacto adicionam novas informações à análise insumo-produto ao incorporar as componentes do valor adicionado à equação básica do modelo. São dois os principais efeitos calculados: efeito direto e efeito indireto.

O efeito direto mede o impacto sobre uma variável em relação a uma situação de alteração na demanda final de uma determinada atividade, considerando apenas as atividades que fornecem insumos diretamente a esta atividade. $O$ efeito indireto mede o impacto sobre uma variável a uma variação na demanda final de uma determinada atividade, considerando todas as atividades que fornecem insumos, direta e indiretamente a essa atividade (GUILHOTO e SESSO FILHO, 2010; FEIJÓ, 2013).

Pela ótica de análise da endogeneização, também é possível verificar os impactos sobre emprego e renda segundo o tipo de multiplicador: o Multiplicador Tipo I considera apenas o fluxo econômico entre os setores (primeira rodada de transações); e, o Multiplicador Tipo II inclui o consumo das famílias nas transações econômicas (segunda rodada de transações) (MILLER e BLAIR, 2009; GUILHOTO, 2011).

\subsection{Multiplicador de renda}

Definido em FEIJÓ (2013), o vetor $W$ de dimensão $n \times 1$, onde $w_{i}$ é a relação entre salário e o valor da produção da atividade $i(i=1,2, \cdots, n)$, tem-se que o valor total dos salários (S) é escrito pela equação (11). 


$$
S=W^{\prime} \times g
$$

Onde,

$$
w_{i}=\frac{s_{i}}{g_{i}}
$$

Sendo $S_{i}=$ salários pagos na atividade $i$ e $g_{i}=$ valor bruto de produção do produto i. Substituindo g por Z x f em (11), obtém-se a equação (13).

$$
S=W^{\prime} \times Z \times f
$$

Onde $\mathrm{Z}$ corresponde a matriz de Leontief e $f=$ corresponde aos itens de demanda final. Nesse caso, a matriz A é chamada de matriz dos coeficientes técnicos diretos e $(\mathrm{I}-\mathrm{A})^{-1}$ a matriz de Leontief ou matriz de coeficientes técnicos diretos mais indiretos.

Desenvolvendo a matriz de Leontief em uma série de potências até a ordem k, com o valor de $k$ dependendo da precisão, tem-se a equação (14).

$$
S=W^{\prime} \times\left(I+A+A^{2}+A^{3}+\ldots+A^{k}\right) \times f
$$

As potências de A, definidas na equação (14), correspondem as diversas rodadas de consumo a partir da estrutura da economia. A partir dessa formulação define-se então a equação (15).

$$
W^{\prime} \times A=\text { multiplicador direto de salário }
$$

Esse multiplicador é interpretado como o impacto de um aumento unitário da demanda final da atividade i sobre os salários pagos pelas atividades que fornecem insumos diretamente para a atividade $i$.

$$
W^{\prime} \times(I-A)^{-1}=\text { multiplicador direto e indireto de salário }
$$

Esse multiplicador é interpretado como o impacto de um aumento unitário da demanda final da atividade i sobre os salários pagos por todas as atividades encadeadas direta e indiretamente com essa atividade.

A mesma formulação pode ser utilizada para calcular multiplicadores para qualquer uma das variáveis que compõem o valor adicionado como os impostos por atividade, ou seja, tem-se três tipos de indicadores para análise de impactos sobre os salários $W$ das atividades que aumentam sua produção; $W^{\prime}$ x $A$ é o impacto nos salários das atividades que fornecem insumos diretamente e; $W^{\prime} \times(I-A)^{-1}$ é 0 impacto nos salários de todas as atividades que são afetadas. 


\subsection{Multiplicador de emprego}

Dispondo do número de pessoas ocupadas em cada atividade é possível calcular o vetor $L$, em que cada componente $l_{i}$ é a relação entre o número de pessoas ocupadas e o valor da produção da atividade i. Assim, o número de pessoas ocupadas pode ser descrito como pela equação (17).

$$
P O=L^{\prime} \times g
$$

Em que,

$$
l_{i}=\frac{P O_{i}}{g_{i}}
$$

e $P O_{i}=$ pessoal ocupado na atividade $i$.

Pode-se então escrever as equações (19) e (20).

$$
L^{\prime} \times A=\text { multiplicador direto de pessoal ocupado }
$$

$$
L^{\prime} x(I-A)^{-1}=\text { multiplicador direto e indireto de pessoal ocupado }
$$

O multiplicador direto pode ser interpretado como o impacto de um aumento unitário da demanda final da atividade i sobre o número de pessoas ocupadas nessa atividade. E o impacto direto mais indireto pode ser interpretado como o impacto de um aumento unitário da demanda final da atividade i sobre o pessoal ocupado por todas as atividades encadeadas direta e indiretamente com essa atividade.

\subsection{Base de dados}

As bases de dados utilizadas neste trabalho foram: a) Tabela de Recursos e Usos do Brasil, ano de 2010, das Contas Nacionais publicadas pelo Instituto Brasileiro de Geografia e Estatística (IBGE); b) Programa de Disseminação das Estatísticas do Trabalho (PDET) do Ministério do Trabalho e Emprego, base estatística da Relação Anual das Informações Sociais (RAIS), dados de Mato Grosso do Sul, estrutura base Setorial CNAE 95 Grupo, com extração de informações do código 011 ao código 926 (224 códigos sequenciais), configurando a extração das informações, para o ano de 2010, de Vínculo Ativo até 31/12 e Valor da Remuneração Média Nominal e; c) Matriz de Insumo-Produto para o estado de Mato Grosso do Sul, contemplando 20 setores de atividades econômicas no Estado, conforme quadro 1. 
Quadro 1. Setores da economia do Mato Grosso do Sul, ano 2010

\begin{tabular}{|ll|}
\hline$N^{\circ}$ & Setores da economia de Mato Grosso do Sul utilizados neste trabalho \\
\hline 1 & Agropecuária \\
3 & Extrativa mineral \\
4 & Têxteis \\
5 & Artigos do vestuário e acessórios \\
6 & Artefatos de couro e calçados \\
7 & Produtos de madeira exclusive móveis \\
8 & Celulose e produtos de papel \\
9 & Jornais, revistas, discos \\
10 & Álcool \\
11 & Produtos químicos \\
12 & Artigos de borracha e plástico \\
13 & Minerais não-metálicos \\
14 & Fabricação de aço e derivados \\
15 & Produtos de metal exclusive máquinas e equipamentos \\
16 & Máquinas e aparelhos de material elétrico \\
17 & Outras indústrias de transformação \\
20 & Serviços industriais de utilidade pública \\
\hline
\end{tabular}

Fonte: Dados da pesquisa.

A escolha dos setores foi realizada mediante a representatividade destes para a economia do Mato Grosso do Sul, e seguiu a ordem aproximada das Contas Nacionais do IBGE.

\section{Resultados e Discussão}

Mensurando, a partir da matriz de transações e da obtenção dos coeficientes técnicos, obteve-se os índices de ligação para trás no modelo aberto da matriz na produção de Mato Grosso do Sul, em que foi verificada a existência de seis setores 
de impacto na incorporação de valor adicionado em termos de compras dentro do Estado (Figura 2).

Figura 2. Índices de ligação para trás segundo a Matriz Insumo-Produto para o Mato Grosso do Sul, 2010, modelo aberto

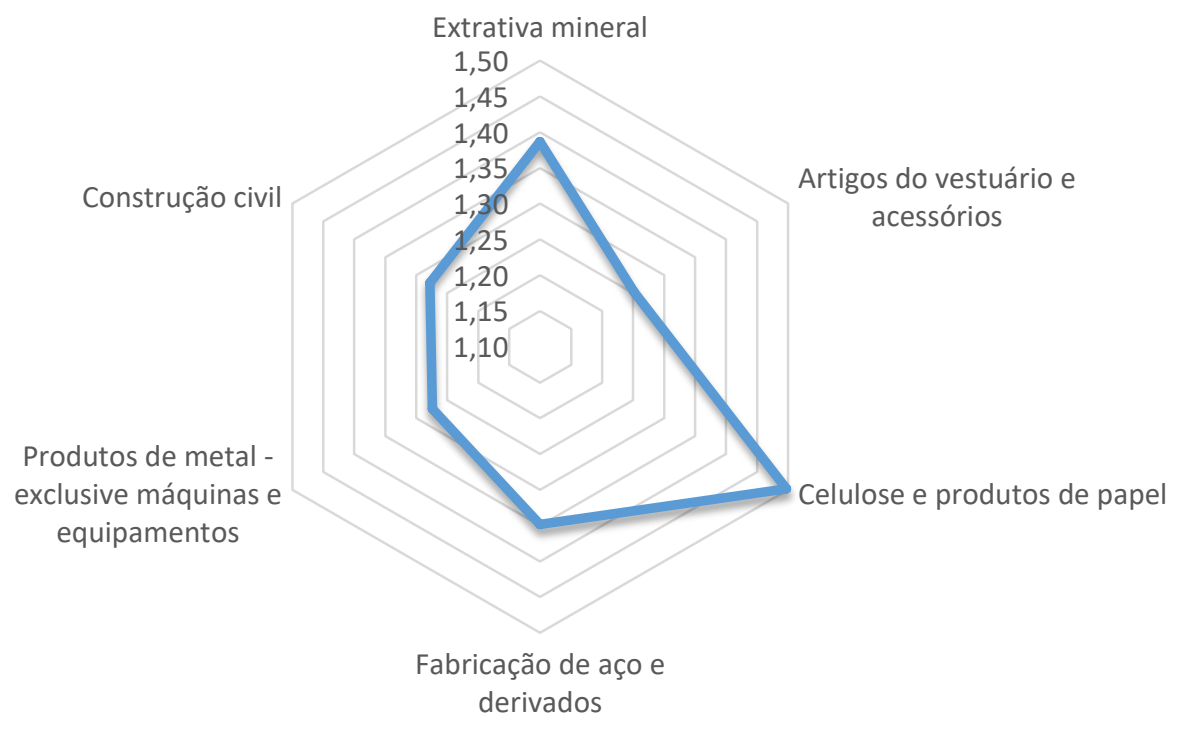

Fonte: Dados da pesquisa.

De acordo com a pesquisa, foi identificado, por exemplo, que a Indústria de Celulose e Produtos de Papel é o setor cuja produção tem maior resposta ao aumento da demanda final da economia, ou seja, com o aumento da demanda de seus produtos finais, este setor é o que mais impacta em termos de compras junto aos demais setores para o desenvolvimento do próprio setor, e a busca de fornecedores dentro do próprio estado, principalmente em função da dinamização local exigida no escoamento de seu processo industrial e ao transporte da madeira.

Segundo informações contidas no Diagnóstico Socioeconômico de Mato Grosso do Sul (SEMADE, 2015), a série histórica do PIB/MS entre 2002 e 2012 demonstra um aumento de participação do setor secundário na formação da riqueza do Estado, passando de 16\% em 2002 para 21\% em 2012. Conforme a figura 2, as atividades Extrativa Mineral, Construção Civil, Produtos de Metal, Fabricação de Aço e Artigos do Vestuário se destacaram no sentido de possuírem os maiores índices de ligação para trás, ou seja, que possuíam o maior impacto na cadeia produtiva.

Castelão et al. (2020) corroboram e apresentam que o setor da indústria de MS possuía importante efeito na economia sul-mato-grossense, em especial, na cadeia produtiva do próprio Estado.

Se for considerada a retroalimentação da matriz de transações e incluir-se o consumo das famílias, obtém-se também seis setores que impactam a economia do estado conforme os índices de ligação para trás, em que verifica-se que os setores Celulose, Extrativa Mineral, Construção Civil, e Artigos do Vestuário, impactam em 
compras aos outros setores tanto somente quando se consideram as transações intersetoriais, mas, também, quando se inserem as rendas das famílias que demandam seus produtos (Figura 3 ).

Figura 3. Índices de ligação para trás segundo a Matriz Insumo-Produto para o Mato Grosso do Sul, 2010, modelo fechado

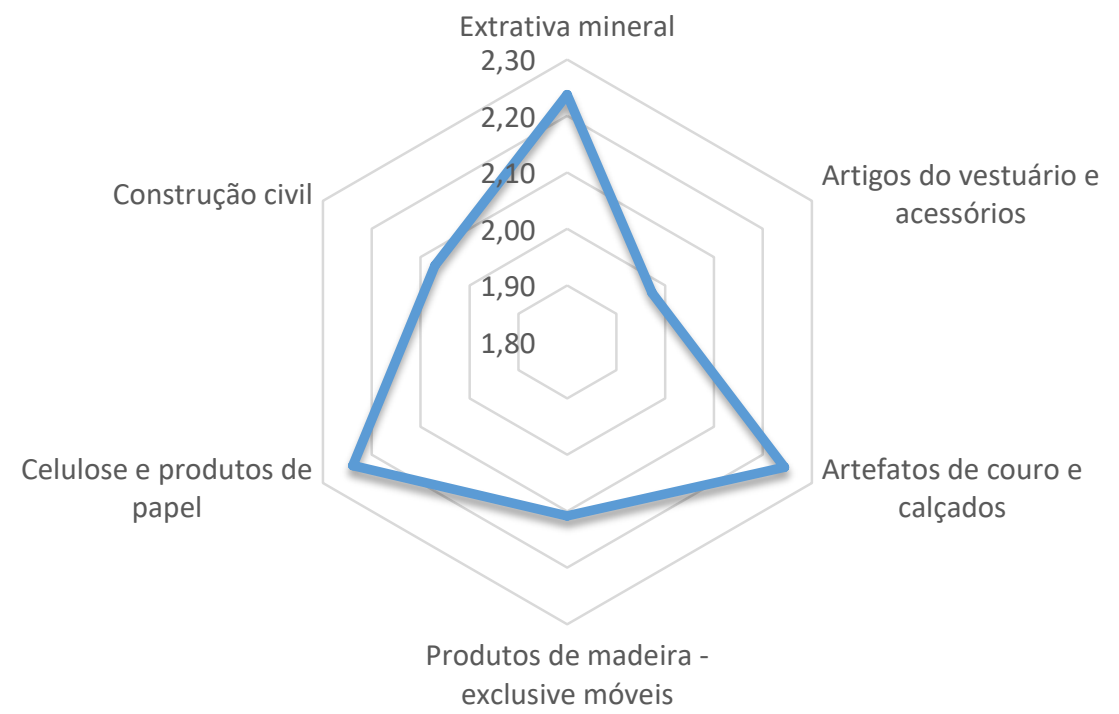

Fonte: Dados da pesquisa.

Essa agregação de valor nos processos produtivos industriais desses setores se deve à política incentivada no Estado, com o objetivo de diversificar a base econômica e promover o incremento da riqueza e da renda através do aumento do emprego e da arrecadação de impostos, em que se busca atrair investimentos de capital privado como forma de dinamizar a economia regional em MS (SEMADE, 2015). Como exemplo, as atividades de produção de celulose - produtos de papel e produtos de plástico-borracha se destacam quando analisados os indicadores de geração de emprego e participação na economia regional (investimentos aportados) (CASTELAO et al., 2020).

Contudo, estimula-se ainda o desenvolvimento industrial voltado para unidades agroindustriais que utilizem como insumos básicos: carne, couro, leite, soja, cereais, cana-de-açúcar, madeira da silvicultura e outros, visando estimular o consumo e a criação de valor adicionado da produção interna, promovendo poder de compra à sua população.

Essa política de estímulo também a agroindústria se deve ao fato de o setor ter participação fundamental e já estabelecida no Estado, chegando a responder por até 37\% do PIB do Mato Grosso do Sul em 2012 (FRAINER et al., 2018).

Com a endogeneização da economia (modelo fechado), se destaca os setores Artefatos de Couro e Produtos de Madeira que, com a inclusão da renda das famílias nas transações econômicas, tem o requerimento de sua demanda 
aumentada, sendo que em MS encontram-se 10 (dez) unidades industriais de beneficiamento do couro até a fase do Wet-Blue que, em seguida, grande parte de sua produção é exportada para a indústria do couro dos estados de São Paulo e Rio Grande do Sul e/ou enviado para mercados externos, como China e Itália, indicando que a obtenção dos suprimentos necessários ao ajuste de sua produção ocorre dentro de Mato Grosso do Sul, de forma que, há um impacto econômico positivo para o Estado (SEMADE, 2015).

Ao considerar os setores que impactam a economia do Estado realizando compras e também vendas, simultaneamente, e incluindo a produção demandada pelas famílias, tem-se três setores-chaves (Figura 4).

Figura 4: Setores-chave segundo índices de ligação da Matriz Insumo-Produto para o Mato Grosso do Sul, 2010, modelo fechado

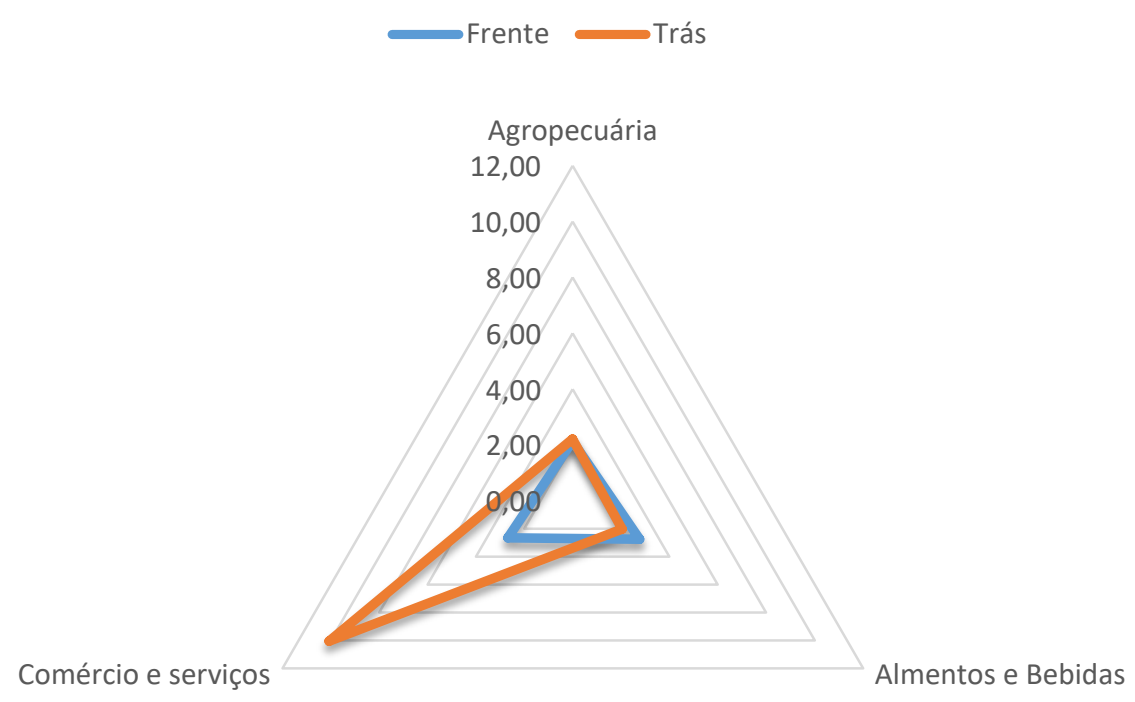

Fonte: Dados da pesquisa.

Esse resultado demonstra a importância do setor de serviços e os relativos à agropecuária na economia sul-mato-grossense que, além de responder por uma importante parcela do PIB estadual, possuem potencial de impacto positivo na economia do Estado conforme aumentam sua produção, realizando compras e escoando também seus resultados dentro do próprio Estado.

O setor de Comércio e Serviços corresponde a 62\% na formação do PIB do MS (IBGE, 2010), demonstrando a relevância das transações econômicas desse setor junto aos demais setores. Já o setor de Alimentos e Bebidas representa $42 \%$ do total da indústria de transformação no Estado, indicando suas trocas comerciais na produção básica do Estado (SEMADE, 2015).

Quanto a Agropecuária, no conjunto da produção das principais lavouras (soja, milho, arroz, trigo, algodão, feijão e sorgo), no ano de 2013, Mato Grosso do Sul se posicionou como o quinto Estado agrícola do país, com uma produção de 13.703 .363 toneladas de grãos, representando $7,3 \%$ da colheita nacional, e, obteve ainda como destaque 42.399.659 toneladas de cana-de-açúcar (quinto produtor nacional) e 721.870 
toneladas de mandioca (décimo produtor nacional) (SEMADE, 2015). A atividade agropecuária do MS apresenta importante efeito nas exportações (ótica da produção), enquanto em relação a renda possui relativa pequena participação de efeito na economia do Mato Grosso do Sul (FAGUNDES et al., 2016).

Em relação à geração de emprego, em 2010, a indústria admitiu 102.732 pessoas e demitiu 94.383, gerando um saldo líquido positivo de 7.989 empregos em MS, saldo superior ao alcançado pelo comércio e a agropecuária que geraram respectivamente 5.042 e 113 colocações líquidas em suas atividades (MTE, 2010). Os impactos dos setores produtivos considerados nesta pesquisa em termos de geração de empregos podem ser visualizados na tabela 1.

Tabela 1. Multiplicador de impacto quanto à geração de emprego para uma variação da demanda final de $\mathrm{R} \$ \mathbf{1 . 0 0 0 . 0 0 0}$ segundo a Matriz Insumo-Produto no Mato Grosso do Sul em 2010, considerando o consumo das famílias

\begin{tabular}{|c|c|c|c|c|}
\hline \multirow{2}{*}{ Setores } & \multicolumn{3}{|c|}{ Multiplicador II } & \multirow[b]{2}{*}{ Induzido } \\
\hline & Direto & Indireto & Total & \\
\hline Agropecuária & 17 & 14 & 31 & 9 \\
\hline Extrativa mineral & 3 & 16 & 20 & 10 \\
\hline Alimentos e bebidas & 6 & 24 & 29 & 10 \\
\hline Têxteis & 16 & 5 & 20 & 4 \\
\hline Artigos do vestuário e acessórios & 37 & 13 & 50 & 8 \\
\hline Artefatos de couro e calçados & 16 & 15 & 31 & 14 \\
\hline Produtos de madeira exceto móveis & 25 & 14 & 40 & 11 \\
\hline Celulose e produtos de papel & 1 & 14 & 15 & 9 \\
\hline Jornais, revistas, discos & 31 & 9 & 40 & 8 \\
\hline Álcool & 5 & 8 & 14 & 4 \\
\hline Produtos químicos & 35 & 1 & 36 & 1 \\
\hline Artigos de borracha e plástico & 7 & 8 & 15 & 5 \\
\hline Minerais não-metálicos & 25 & 11 & 36 & 9 \\
\hline Fabricação de aço e derivados & 2 & 7 & 8 & 3 \\
\hline $\begin{array}{l}\text { Produtos de metal exceto máquinas e } \\
\text { equipamentos }\end{array}$ & 1 & 8 & 9 & 5 \\
\hline Máquinas e aparelhos elétricos & 12 & 1 & 14 & 1 \\
\hline Outras indústrias de transformação & 6 & 10 & 17 & 7 \\
\hline Serviços industriais de utilidade pública & 2 & 8 & 9 & 7 \\
\hline Construção civil & 22 & 14 & 35 & 9 \\
\hline Comércio e serviços & 23 & 22 & 45 & 16 \\
\hline
\end{tabular}

Fonte: Dados da pesquisa.

Analisando o multiplicador de impacto quanto à geração de empregos nas relações intersetoriais do MS, considerando o consumo das famílias na matriz de transações econômicas, conforme tabela 1, verifica-se que a cada $\mathrm{R} \$ 1.000 .000,00$ aplicados na demanda final do setor de Artigos do Vestuário e Acessórios, tem-se a geração de 50 (cinquenta) empregos totais, o que reflete o fato de que nesse setor estão concentrados 11\% dos estabelecimentos industriais no Estado (SEMADE, 2015). 
Ao se considerar a diferença entre geração de empregos somente nas relações intersetoriais e quanto à endogeneização da economia, tem-se o acréscimo da criação de mais postos de trabalho, em que o setor de Comércio e Serviços se destaca com 16 (dezesseis) empregados a mais contratados no Estado (Emprego Induzido).

A tabela 2 apresenta o multiplicador de renda (salários) no estado de Mato Grosso do Sul, em que se aponta que a cada $\mathrm{R} \$ 1.000$ aplicados na demanda final do setor de Comércio e Serviços este gera um impacto de aumento de renda direta em forma de salários de $R \$ 296$ e de renda indireta de $R \$ 279$, totalizando $R \$ 575$ de aumento de renda para economia local.

Tabela 2. Multiplicador de impacto quanto à geração renda para uma variação da demanda final de $\mathrm{R} \$ 1.000$ segundo a Matriz Insumo-Produto no Mato Grosso do Sul em 2010, considerando o consumo das famílias

\begin{tabular}{lcccc}
\hline \multirow{2}{*}{ Setores } & \multicolumn{3}{c}{ Multiplicador II } & \multirow{2}{*}{ Induzido } \\
\cline { 2 - 4 } & Direto & Indireto & Total & \\
\hline Agropecuária & 161 & 165 & 325 & 112 \\
Extrativa mineral & 149 & 214 & 363 & 126 \\
Alimentos e bebidas & 77 & 281 & 358 & 124 \\
Têxteis & 99 & 57 & 156 & 54 \\
Artigos do vestuário e acessórios & 155 & 153 & 308 & 106 \\
Artefatos de couro e calçados & 311 & 188 & 499 & 173 \\
Produtos de madeira excl. móveis & 229 & 174 & 403 & 139 \\
Celulose e produtos de papel & 125 & 191 & 316 & 109 \\
Jornais, revistas, discos & 182 & 118 & 301 & 104 \\
Álcool & 59 & 98 & 158 & 55 \\
Produtos químicos & 17 & 16 & 33 & 11 \\
Artigos de borracha e plástico & 78 & 102 & 180 & 62 \\
Minerais não-metálicos & 173 & 141 & 314 & 109 \\
Fabricação de aço e derivados & 14 & 99 & 113 & 39 \\
Produtos metal fora máquinas equipamentos & 89 & 104 & 193 & 67 \\
Máquinas e aparelhos de material elétrico & 12 & 13 & 26 & 9 \\
Outras indústrias de transformação & 113 & 134 & 247 & 86 \\
Serviços Industriais de Utilidade Pública & 151 & 105 & 256 & 89 \\
Construção civil & 174 & 164 & 338 & 117 \\
Comércio e serviços & 296 & 279 & 575 & 199 \\
\hline Fonte: Dados da pesquisa. & & & 59 \\
\hline
\end{tabular}

Fonte: Dados da pesquisa.

Além disso, o setor também impacta a economia de forma induzida, acrescentando $\mathrm{R} \$ 199$ de geração de renda ao se considerar o poder de compra das famílias nas transações econômicas do Estado (Renda Induzida).

Os resultados derivados da matriz insumo-produto, tanto o efeito para frente quanto para trás em relação ao encadeamento produtivo e seus impactos, possuem similaridade com dados de outras pesquisas, principalmente, no que tange 
aos efeitos indiretos do setor secundário e a importância do agronegócio enquanto importante demandador (CASTELAO et al., 2020).

\section{Conclusão}

Os resultados encontrados refletem a economia do estado de Mato Grosso do Sul em termos do agregado macroeconômico e via relações intersetoriais do modelo de insumo-produto. Conforme descrito no objetivo do trabalho, os encadeamentos das atividades ficaram melhor delineados, possibilitando uma melhor percepção da importância relativa das atividades econômicas no Estado. A determinação dos multiplicadores de emprego e renda bem como dos indicadores de síntese podem servir de instrumento para a tomada de decisão dos agentes públicos e de diversas instituições privadas.

Referente às relações intersetoriais, para uma matriz aberta, foram identificados seis setores de destaque em termos de encadeamento para trás: Extrativa Mineral, Artigos do Vestuário e Acessórios, Celulose e Produtos de Papel, Fabricação de Aço e Derivados, Produtos de Metal, e, Construção Civil.

Em se considerando a renda das famílias nas transações econômicas, incluem-se os setores Artefatos de Couro e Calçados e Produtos de Madeira, com relevância nas compras junto aos demais setores produtivos dentro do Estado.

Também, os setores Agropecuária, Alimentos e Bebidas e Comércio e Serviços, impactam para frente e para trás, simultaneamente, ou seja, adquirindo seus insumos de produção e escoando seus resultados produtivos dentro do próprio Mato Grosso do Sul.

Em termos de impactos sobre o emprego e renda, os setores que mais se destacam são Comércio e Serviços, Produtos de Madeira, Artefatos de Couro e Caçados, Alimentos e Bebidas, Artigos do Vestuário e Acessórios, e, Extrativa Mineral.

Os resultados e as informações obtidas neste estudo permitem disponibilizar, para os pesquisadores, autoridades políticas e econômicas e também para os demais interessados, um conjunto de informações que contribuem para se avançar em estudos específicos associados aos modelos de insumo-produto, notadamente aqueles direcionados ao planejamento do desenvolvimento regional sustentável.

\section{REFERÊNCIAS}

BÊRNI, D. A.; LAUTERT, V. Mesoeconomia: lições de contabilidade social: a mensuração do esforço produtivo da sociedade. Porto Alegre: Bookman, 2011.

BUNGENSTAB, D. J. Agronegócio com sustentabilidade: a eficiência das cadeias produtivas do agronegócio em Mato Grosso do Sul. Brasília, DF: Embrapa, 2012.

CASTELAO, R. A.; SOUZA, C. C.; FRAINER, D. M. Aplicações da Matriz Insumo-Produto em análises ambientais no Brasil: uma revisão sistemática de literatura. Ciência e Natura, v. 41, p. 1-10, 2019. 
CASTELAO, R. A.; SOUZA, C. C.; FRAINER, D. M.; CARNEIRO JUNIOR, J. B. A. Crescimento econômico e indução de alteração ambiental no Mato Grosso do Sul entre 1991 e 2010.

Revista Ibero-americana de Ciências Ambientais, v. 11, p. 331-344, 2020.

CASTELANI, S. A.; GUILHOTO, J. J. M.; IGLIORI, D. A. Local Demand, Urbanization and Amazonian Metropolitan Regions Impacts on Deforestation of the Brazilian Amazon. 53rd Congress of the European Regional Science Association, Palermo, Italy - August 27th to 31st, 2013.

COSTA, C. C.; GUILHOTO, J. J. M.; IMORI, D. Importância dos Setores Agroindustriais na Geração de Renda e Emprego para a Economia Brasileira. Revista de Economia e Sociologia Rural, Piracicaba-SP, Vol. 51, № 4, p. 791-808, Out/Dez 2014.

COUTINHO, M.; BITENCOURT, M.; FIGUEIREDO-NETO, L.; FIGUEIREDO, L. A contribuição das atividades de base agropecuária na geração de emprego nos municípios de Mato Grosso do Sul (Brasil). Revista Latinoamericana de Estudios Urbano-Regionales, v. 45, n. 135, p. 223244, 2019.

FAGUNDES, M. B. B.; DIAS, D. T.; FIGUEIREDO, A. M. R.; PITALUGA, C. M. interligações setoriais e composição do produto, demanda e renda da agropecuária de Mato Grosso do Sul: uma aplicação da matriz insumo-produto. CONTEXTUS Revista Contemporânea de Economia e Gestão, v. 14, n. 1, jan/abr 2016.

FEIJÓ, C. A.; RAMOS, R. L. O. Contabilidade Social: A Nova Referência das Contas Nacionais do Brasil. 4 ed. rev. e atual. Rio de Janeiro: Elsevier Campus, 2013.

FIGUEIREDO, N. R. M. Construção da matriz de insumo-produto híbrida para o estado de Pernambuco e avaliação da intensidade energética e de emissões de $\mathrm{CO} 2$ setorial. $72 \mathrm{f}$. Dissertação (Programa de Pós-Graduação em Economia) - Universidade Federal da Paraíba, Paraíba, 2009.

FRAINER, D. M.; SOUZA, C. C.; REIS NETO, J.F.; CASTELAO, R. A.; ROSA, M. Mensuração do Produto Interno Bruto do Agronegócio de Mato Grosso do Sul. Revista de ciências agrárias (LISBOA), v. 41, p. 1135-1145, 2018.

GUILHOTO J. J. M. A Model for Economic Planning and Analysis for the Brazilian Economy. Dissertação de Doutorado. University of Illinois at Urbana-Champaign (EUA), 1986.

GUILHOTO, J. J. M.; SESSO, U. A. F. Desenvolvimento Econômico e Regional: Estimação da Matriz Insumo-Produto Utilizando Dados Preliminares das Contas Nacionais. Economia e Tecnologia, São Paulo, v. 4, n. 23, p. 53-62, 2010.

GUILHOTO, J. J. M.; SESSO FILHO, U. A.; LOPES, R. L.; HILGEMBERG, C. M. A. T.; HILGEMBERG, E. M. Nota metodológica: construção da matriz insumo-produto utilizando dados preliminares das contas nacionais. In: II Encontro de Estudos Regionais e Urbanos, 10, 2002, São Paulo. Anais... São Paulo: USP, p. 25-37, 2002.

GUILHOTO, J. J. M. Input-Output Analysis: Theory and Foundations. Núcleo de Economia Regional e Urbana da Universidade de São Paulo (NEREUS), 2011. 
GONÇALVES JUNIOR, C. A.; GUILHOTO, J. J. M. Productive Structure and Trade Relations: The Case of the Western Border Regions of Paraná State, Brazil. 22nd International InputOutput Conference Lisbon, Portugal, July 15-18, 2014.

HADDAD, E. A.; GONÇALVES JÚNIOR, C. A.; NASCIMENTO, T. O. Matriz interestadual de insumo-produto para o brasil: uma aplicação do método IIOAS. Revista Brasileira de Estudos Regionais e Urbanos, v. 11, n. 4, p. 424-446, 12 fev. 2018.

HILGEMBERG, E. M. Quantificação e efeitos econômicos do controle de emissões de $\mathrm{CO}_{2}$ decorrentes do uso de gás natural, álcool e derivados de petróleo no Brasil: um modelo interregional de insumo-produto. 16of. Tese (Doutorado em Ciências) - Escola Superior de Agricultura Luiz de Queiroz, Universidade de São Paulo, Piracicaba, 2004.

HIRSCHMAN, A. O. The strategy of economic development. New Haven: Yale University Press, 1958.

IBGE. Instituto Brasileiro de Geografia e Estatística. Confronto dos resultados dos dadosestruturais dos Censos Agropecuários Mato Grosso do Sul: 1970/2006, 2016.

IBGE. Instituto Brasileiro de Geografia e Estatística. Contas Regionais do Brasil 2010: tabelas completas 2002 2010, 2016.

IBGE (2015). Instituto Brasileiro de Geografia e Estatística. Contas Nacionais Brasil 2005:2009. Tabelas de Recursos e Usos, 2015.

LEONTIEF, W. W. A economia do insumo-produto. 2ed. São Paulo: Nova Cultural, 1986.

LEONTIEF, W. W. Quantitative Input and Output Relations in the Economic Systems of the United States. The Review of Economics and Statistics, Cambridge. 1936; v. 18, n. 3, p. 105125.

MILLER, R. E.; BLAIR, P. D. Input-Output Analysis: Foundations and Extensions. 2ed. Cambridge: University Press, 2009.

MIRANDA, C. R. Economia e meio ambiente: uma abordagem de insumo-produto. Pesquisa e planejamento econômico. 1980; Rio de Janeiro, v 10, n. 2, p. 601-636.

MTE. Ministério do Trabalho e Emprego. Programa de Disseminação das Estatísticas do Trabalho (PDET). Base estatística Relação Anual das Informações Sociais (RAIS), 2014.

MONTOYA, M. A. A inserção insumo-produto da economia brasileira no Mercosul: uma abordagem pelo valor adicionado. Revista Brasileira de Economia, Rio de Janeiro, v. 55, n. 2, p. 253-282, Apr. 2001.

MONTOYA, M. A.; PASQUAL, C. A.; LOPES, R. L.; GUILHOTO, J. M. Consumo de Energia, Emissões de $\mathrm{CO}_{2}$ e a Geração de Emprego e Renda no Agronegócio Brasileiro: Uma Análise Insumo-Produto. Economia aplicada, São Paulo, v. 20, n. 4, p. 338-412, 2013.

MONTEIRO, D. C. REJANI, F. C. LOPES, R. L. RODRIGUES, R. L. EMISSÕES DE CO2 E CONSUMO DE ENERGIA NO PARANÁ: UMA ABORDAGEM INSUMO-PRODUTO. Revista Brasileira de Estudos Regionais e Urbanos, vol. 06, n. 2, pp. 19-35, 2012. 
RASMUSSEN, P. N. Studies in intersectoral relations. Amsterdam: North-Holland, 1956.

SALVO, G.; SIMAS, M. S.; PACCA, S. A.; GUILHOTO, J. J. M.; TOMAS, A. R. G.; ABRAMOVAY, R. Estimating the human appropriation of land in Brazil by means of an Input-Output Economic Model and Ecological Footprint analysis. Ecological Indicators, London, v. 53, n. 4, p. 78-94, 2015.

SEMADE. Secretaria de Estado de Meio Ambiente e Desenvolvimento Econômico. Diagnóstico Socioeconômico de Mato Grosso do Sul 2015. Disponível em: http://www.semade.ms.gov.br/1497-2/. Acesso em 20 de novembro de 2018.

THOMAS, J. M.; CALLAN, S. J. Economia ambiental: aplicações, política e teoria. São Paulo: Cengage Learning, 2010.

Edrilene Barbosa Lima Justi. Doutora em Meio Ambiente e Desenvolvimento Regional. UFMS. Professora Adjunta da UFMS, Campus de Nova Andradina, do curso de Administração. Universidade Federal de Mato Grosso do Sul, CPNA, Nova Andradina, MS - Brasil - Caixa-postal: 128. edrilene@gmail.com

Raul Asseff Castelao. Doutor em Meio Ambiente e Desenvolvimento Regional. Uniderp. Avenida Alexandre Herculano, 1400, Jardim Veraneio, Campo GrandeMS.raulassefcastelao@gmail.com

Celso Correia de Souza. Doutor em Engenharia Elétrica. Uniderp. Professor titular do programa de pós graduação em Meio Ambiente e Desenvolvimento Regional Avenida Alexandre Herculano, 1400, Jardim Veraneio, Campo GrandeMS.csousa939@gmail.com

Daniel Massen Frainer. Doutor em Economia. UEMS. Professor Associado da Universidade Estadual de Mato Grosso do Sul. Avenida Alexandre Herculano, 1400, Jardim Veraneio, Campo Grande-MS. danielfrainer@gmail.com

Como citar: JUSTI, Edrilene Barbosa Lima et al. Desenvolvimento de Mato Grosso do Sul: uma análise dos setores produtivos do estado a partir do modelo insumo-produto. Redes (St. Cruz Sul, Online), Santa Cruz do Sul, v. 25, p. 2385-2406, 2020. ISSN 1982-6745. doi:https://doi.org/10.17058/redes.v25io.14740. 
Desenvolvimento de Mato Grosso do Sul: uma análise dos setores produtivos do estado a partir do modelo insumo-produto

\section{CONTRIBUIÇÃO DE CADA AUTOR}

a. Fundamentação teórico-conceitual e problematização: Edrilene, Raul, Celso e Daniel

b. Pesquisa de dados e análise estatística: Daniel e Edrilene

c. Elaboração de figuras e tabelas: Edrilene, Raul, Celso e Daniel

d. Fotos: Não se aplica.

e. Elaboração e redação do texto: Edrilene, Raul, Celso e Daniel

f. Seleção das referências bibliográficas: Edrilene, Raul, Celso e Daniel

Fontes de financiamento: Não se aplica. 